\title{
Angiogenin is upregulated during the alloreactive immune response and has no effect on the T-cell expansion phase, whereas it affects the contraction phase by inhibiting $\mathrm{CD4}^{+} \mathrm{T}$-cell apoptosis
}

\author{
THEODOROS ELEFTHERIADIS $^{1}$, GEORGIOS PISSAS $^{1}$, MARIA SOUNIDAKI $^{1}$, \\ NIKOLAOS ANTONIADIS ${ }^{2}$, GEORGIA ANTONIADI ${ }^{1}$, VASSILIOS LIAKOPOULOS ${ }^{1}$ and IOANNIS STEFANIDIS ${ }^{1}$ \\ ${ }^{1}$ Department of Nephrology, University of Thessaly Medical School, 41110 Larissa; ${ }^{2}$ Organ Transplant Unit, \\ Hippokration General Hospital, Aristotle University of Thessaloniki Medical School, 54642 Thessaloniki, Greece
}

Received May 11, 2016; Accepted August 5, 2016

DOI: $10.3892 / \mathrm{etm} .2016 .3786$

\begin{abstract}
Under growth conditions, angiogenin is translocated into the nucleus, where it enhances ribosomal RNA transcription, facilitating increased protein synthesis and cellular proliferation. During stress conditions, angiogenin is sequestered in the cytoplasm, where it cleaves transfer RNA (tRNA) to produce tRNA-derived, stress-induced small RNAs (tiRNAs) that inhibit global protein synthesis, but increase the translation of anti-apoptotic factors. In the present study, the role of angiogenin in the human alloreactive immune response was evaluated using mixed lymphocyte reactions (MLRs) and neamine, an inhibitor of angiogenin nuclear translocation. In MLRs, angiogenin production was significantly $(\mathrm{P}<0.001)$ increased compared with resting peripheral blood mononuclear cells. The addition of neamine had no effect on cell proliferation, but did significantly $(\mathrm{P}<0.001)$ increase expression of $\mathrm{Bcl}-2$-associated $\mathrm{X}$ protein and protein levels of activated caspase- 3 in $\mathrm{CD} 4^{+} \mathrm{T}$-cells isolated from the MLRs, indicating that angiogenin reduces apoptosis. In conclusion, angiogenin is upregulated during the alloreactive immune response, in which it does not affect the T-cell expansion phase, but inhibits the T-cell contraction phase by protecting against $\mathrm{CD} 4^{+} \mathrm{T}$-cell apoptosis.
\end{abstract}

\section{Introduction}

Angiogenin, also known as ribonuclease 5 , is a $14 \mathrm{kDa}$ protein expressed in various tissues that can induce or inhibit target protein synthesis. Under cellular growth conditions angiogenin is translocated into the nucleus where it enhances

Correspondence to: Dr Theodoros Eleftheriadis, Department of Nephrology, University of Thessaly Medical School, Neo Ktirio, Mezourlo Hill, 41110 Larissa, Greece

E-mail: teleftheriadis@yahoo.com

Key words: angiogenin, cluster of differentiation 4 positive T-cells, proliferation, apoptosis ribosomal RNA (rRNA) transcription and thus subsequent ribosome formation and protein synthesis required for cell growth and proliferation $(1,2)$. Under cellular stress conditions, angiogenin is sequestered in the cytoplasm, where it cleaves transfer RNA (tRNA) to produce tRNA-derived, stress-induced small RNAs (tiRNAs). In turn, tiRNAs repress global translation by interfering with translation initiation factors, but specifically facilitate the translation of mRNA that contains internal ribosomal entry sites (IRES) (3-5). Since numerous IRES-containing mRNAs encode pro-survival and anti-apoptotic genes, angiogenin protects cells from apoptosis during cell stress $(6,7)$. In addition, the direct interaction of angiogenin with p53 (8), and interaction between tiRNAs and cytochrome $c$ released from the mitochondria (9), have been detected to inhibit cell apoptosis.

Interestingly, angiogenin may serve a role in the immune response. Angiogenin is expressed in numerous malignant and normal cells, including lymphocytes (10). Angiogenin levels are increased in inflammatory conditions $(11,12)$ and autoimmune diseases, where it has been detected in the synovial fluid of patients with rheumatoid arthritis (13). During the adaptive immune response, the expansion phase (rapid T-cell proliferation) is followed by the contraction phase (T-cell apoptosis). Insufficient apoptosis of activated T-cells can lead to autoimmune diseases, whereas excessive apoptosis can result in immunodeficiency (14). Although angiogenin has been implicated in cell proliferation and apoptosis, its role in the T-cell response has not been well studied.

In the present study, the two-way mixed lymphocyte reaction (MLR) was used as a measure of alloreactivity (15), along with neamine that inhibits the nuclear translocation of angiogenin. Neamine is a neomycin derivative with low toxicity, which represses rRNA transcription and cell proliferation through inhibiting angiogenin nuclear translocation (2,16-18). In addition, nuclear translocation of angiogenin may be required for its ribonuclease activity. In S28N mutant angiogenin that is found in some patients with amyotrophic lateral sclerosis, the mutation is near to the nuclear localization sequence and inhibits nuclear translocation and tiRNA production (19). 


\section{Materials and methods}

Study participants. Blood samples were collected on the same day in February 2016 from 5 healthy volunteers ( 3 men and 2 women; mean age, $36 \pm 8$ years) at the University Hospital of Larissa (Larissa, Greece). Informed consent was obtained from each participant enrolled in the study and the Ethics Committee of the University Hospital of Larissa (Larissa, Greece) approved the study protocol.

Cell culture conditions and two-way MLRs. Peripheral blood mononuclear cells (PBMC) were isolated from whole blood samples by Ficoll-Hypaque density gradient centrifugation using Histopaque-1077 (Sigma-Aldrich; Merck Millipore, Darmstadt, Germany). Briefly, the interface was collected and washed with Roswell Park Memorial Institute (RPMI)-1640 medium (Sigma-Aldrich; Merck Millipore). Then, isolated PBMCs were counted using a Neubauer chamber (Paul Marienfeld $\mathrm{GmbH}$, Lauda-Königshofen, Germany) on an optical microscope. Cell viability was assessed using the trypan blue exclusion assay (Sigma-Aldrich; Merck Millipore). Cells were cultured in RPMI-1640 medium, supplemented with L-glutamine, 10 mM 4-(2-hydroxyethyl)-1-piperazineethanesulfonic acid (HEPES), 10\% fetal bovine serum (Sigma-Aldrich; Merck Millipore) and antibiotic-antimycotic solution (Sigma-Aldrich; Merck Millipore). Cultures were incubated at $37^{\circ} \mathrm{C}$ in an atmosphere of $95 \%$ relative humidity and $5 \% \mathrm{CO}_{2}$.

To determine cell proliferation and neamine cytotoxicity, 10 two-way MLRs were performed in the presence or absence of $100 \mu \mathrm{M}$ neamine (angiogenin nuclear translocation inhibitor; Sigma-Aldrich; Merck Millipore). This concentration was chosen as it was in line with previous experiments $(17,18)$. MLRs were performed in 96-well plates over 7 days. The quantity of PBMCs, from each of the two individuals that contribute to the formation of an MLR couple, was $5 \times 10^{4}$ cells measuring $1 \times 10^{5}$ cells per well. Resting (unstimulated) PMBC cultures of $1 \times 10^{5}$ cells per well from each member of the MLR couple were used as controls.

To assess angiogenin concentration in the supernatant of the MLRs and expression of specific proteins (described below) in cluster of differentiation 4 positive $\left(\mathrm{CD}^{+}\right) \mathrm{T}$-cells, 10 two-way MLRs were performed in 12-well plates over 7 days. The number of PBMCs, from each of the two individuals that contribute to the formation of an MLR couple, was $5 \times 10^{5}$ cells, measuring $1 \times 10^{6}$ PBMCs per well. Resting PMBC cultures of $1 \times 10^{6}$ cells per well from each member of the MLR couple were used as controls. At the end of the 7-day period, supernatants were collected and stored at $-80^{\circ} \mathrm{C}$ and $\mathrm{CD} 4^{+}$ T-cells were isolated by negative selection using the Human $\mathrm{CD}^{+}{ }^{+} \mathrm{T}$ Cell Isolation Kit (cat. no. 130-096-533; Miltenyi Biotec GmbH, Bergisch Gladbach, Germany).

Cell proliferation in the MLR. To assess cell proliferation the Cell Proliferation ELISA kit (cat. no. 11647229001; Roche Diagnostics, Indianapolis, IN, USA) was used, which is based on bromodeoxyuridine labeling and immunoenzymatic detection. The proliferation index of cells was calculated as the ratio of the optical density (OD) at $450 \mathrm{~nm}$ of the MLR to the mean OD of the control resting PBMC cultures of the two subjects that constituted each specific MLR. Experiments were performed in triplicate and the results are presented as the mean.

Neamine cytotoxicity. Neamine cytotoxicity was assessed by performing a lactate dehydrogenase (LDH) release assay using the CytoTox Non-Radioactive Cytotoxic Assay kit (cat. no. G1780; Promega Corporation, Madison, WI, USA) in MLRs. To assess cytotoxicity in resting PBMCs, the mean of measurements from the control resting PBMC cultures of the two subjects that constituted each MLR couple were used. Results were expressed as the percentage of dead cells. Experiments were performed in triplicate and the results are presented as the mean.

Angiogenin expression in the MLR. Angiogenin expression in the supernatants of MLRs was assessed by measuring its concentration using an ELISA with a sensitivity of $<6 \mathrm{pg} / \mathrm{ml}$ (cat. no. DAN00; Human Angiogenin Quantikine ELISA; R\&D Systems, Inc., Minneapolis, MN, USA). To assess angiogenin concentration in resting PBMCs, the mean of the measurements derived from the control resting PBMC cultures of the two subjects that constituted each specific MLR couple were used.

Western blotting for expression of specific proteins in MLR $C D 4^{+} T$-cells. The expression of B-cell lymphoma 2 (Bcl-2), $\mathrm{Bcl}-2$-associated $\mathrm{X}$ protein (Bax), activated cleaved caspase-3 (CC3; activated by cleavage at aspartate 175) and $\beta$-actin in $\mathrm{CD}^{+}{ }^{+} \mathrm{T}$-cells isolated from the MLRs was assessed by western blotting. Isolated $\mathrm{CD} 4^{+} \mathrm{T}$-cells were counted using an optical microscope and Neubauer chamber, and cell viability was determined by the trypan blue exclusion assay (Sigma-Aldrich; Merck Millipore). Equal numbers of CD4 ${ }^{+}$ T-cells from each MLR were lysed using the T-PER tissue protein extraction reagent (Thermo Fisher Scientific, Inc., Rockford, IL, USA), supplemented with protease inhibitors [4-(2-aminoethyl)benzenesulfonyl fluoride, E-64, bestatin, leupeptin, aprotinin, phenylmethanesulfonyl fluoride and ethylenediaminetetraacetic acid] and phosphatase inhibitors against acid and alkaline phosphatases (both Sigma-Aldrich; Merck Millipore), in addition to serine/threonine and tyrosine protein phosphatases inhibitors (both Roche Diagnostics). Extracted protein was quantified via the Bradford assay (reagent provided by Sigma-Aldrich; Merck Millipore) and $10 \mu \mathrm{g}$ from each sample was used for SDS-PAGE (4-12\% gels) and subsequent western blotting. Polyvinylidene fluoride (PVDF) blots were incubated at $4^{\circ} \mathrm{C}$ with a primary antibody [rabbit monoclonal anti-Bcl-2 (cat. no. 4223; 1:1,000), rabbit monoclonal anti-Bax (cat. no. 5023; 1:1,000), rabbit monoclonal anti-caspase-3 (activated by cleavage at aspartate 175; cat. no. $9664 ; 1: 1,000)$ or rabbit monoclonal anti- $\beta$-actin (cat. no. 4967; 1:2,500) (all from Cell Signaling Technology, Inc., Danvers, MA, USA)] for $16 \mathrm{~h}$, followed by 3 washes and incubation with a secondary antibody (anti-rabbit IgG, HRP-linked antibody; cat. No. 7074; 1:1,000; Cell Signaling Technology, Inc.) for $30 \mathrm{~min}$ at room temperature. The BenchMark Pre-Stained Protein Ladder (Thermo Fisher Scientific, Inc.) was used as a marker. Bands were then visualized by enhanced chemiluminescent detection using the LumiSensor Plus Chemiluminescent HRP Substrate kit (cat. no. L00225; 


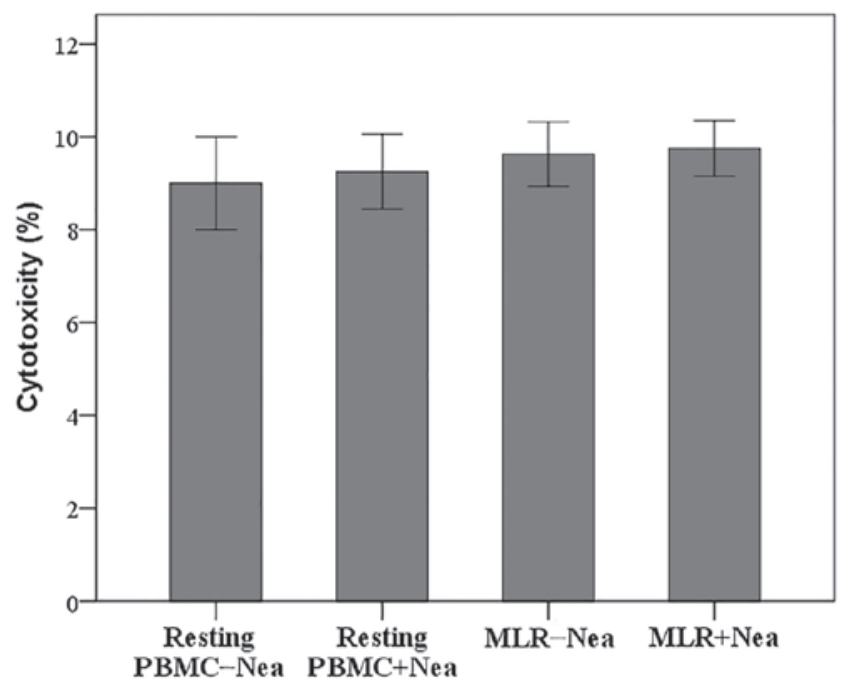

Figure 1. Effect of Nea on cell necrosis. Nea was not cytotoxic to resting PBMCs or MLRs. Ten different MLRs were performed. Results are presented as the mean \pm standard deviation of triplicate readings of the percentage of cell death. PBMC, peripheral blood mononuclear cells; Nea, neamine; MLR, mixed lymphocyte reaction.

GenScript, Piscataway, NJ, USA). When re-probing PVDF blots, the previous antibodies were removed using the Restore Western Blot Stripping Buffer (Thermo Fisher Scientific, Inc.) according to the manufacturer's protocol and western blotting was resumed as previously described, using a different primary antibody.

Statistical analysis. SPSS software (version 13.0; SPSS, Inc., Chicago, IL, USA) was used for statistical analysis. The normality of the evaluated variables was assessed and confirmed by one-sample Kolmogorov-Smirnov tests. For statistical comparison of means a paired-sample t-tests were used. Results are expressed as mean \pm standard deviation. $\mathrm{P}<0.05$ was considered to indicate a statistically significant difference.

Analysis and quantification of the westerns blots was performed using Image $\mathbf{J}$ software (version 1.49; National Institutes of Health, Bethesda, MD, USA) and results expressed as arbitrary OD units. Statistical analysis following normalization for the control OD values was avoided, in order to prevent disruption of the prerequisite for normal distribution of the compared variables when applying parametric statistical tests. However, for the reader's convenience, the results are presented following normalization to the control group.

\section{Results}

Neamine is not cytotoxic to resting PBMCs or MLRs. The $\mathrm{LDH}$ release assay revealed that $100 \mu \mathrm{M}$ neamine does not induce cell necrosis in resting PBMCs or MLRs. Cytotoxicity was $9.00 \pm 1.00 \%$ in resting PBMCs vs. $9.25 \pm 0.80 \%$ in resting PBMCs treated with neamine $(\mathrm{P}=0.692$; Fig. 1). In untreated MLRs cytotoxicity was $10.00 \pm 1.00 \%$ vs. $10.00 \pm 0.75 \%$ in neamine-treated MLRs $(\mathrm{P}=1.000$; Fig. 1). Results are presented as the percentage of cell death.

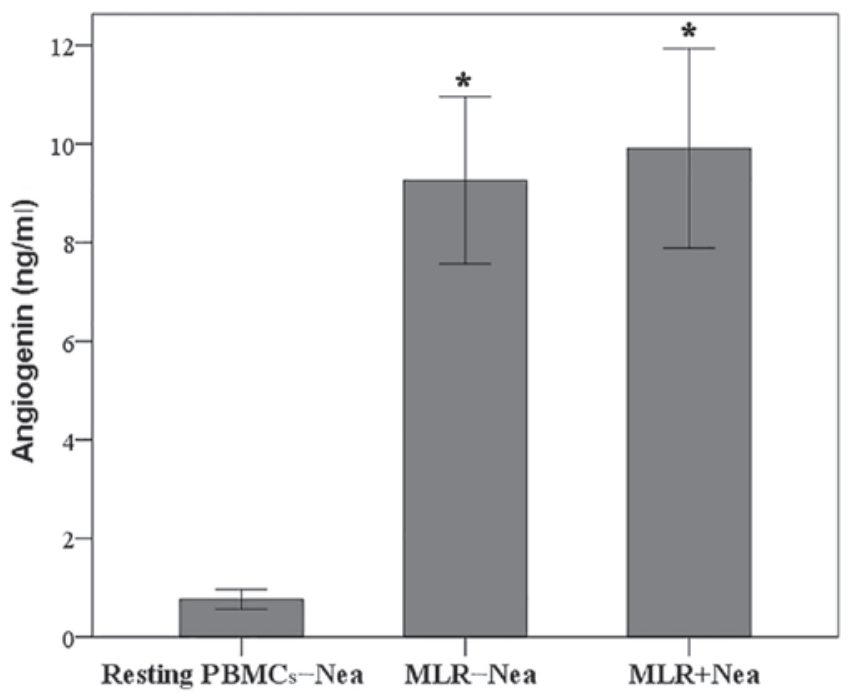

Figure 2. Angiogenin expression in MLR in the presence or absence of Nea. Compared with resting PBMCs, expression of angiogenin in MLRs was significantly higher. Nea did not affect angiogenin expression in MLRs. Ten different MLRs were performed. Results are presented as the mean \pm standard deviation. ${ }^{*} \mathrm{P}<0.001$ vs. resting PMBCs. PBMC, peripheral blood mononuclear cells; MLR, mixed lymphocyte reaction; Nea, neamine.

In MLRs angiogenin expression increased significantly compared with resting PMBCs irrespective of the presence of neamine. Compared to resting PBMCs, angiogenin concentration was significantly increased in the supernatants of MLRs $(0.76 \pm 0.20$ vs. $9.26 \pm 1.69 \mathrm{ng} / \mathrm{ml}$, respectively; $\mathrm{P}<0.001$; Fig. 2). The concentration of angiogenin in the supernatant of neamine-treated MLRs was $9.91 \pm 2.02 \mathrm{ng} / \mathrm{ml}$, significantly higher compared with resting PBMCs $(0.76 \pm 0.20 \mathrm{ng} / \mathrm{ml}$; $\mathrm{P}<0.001$; Fig. 2), but similar to the concentration found in untreated MLRs $(9.26 \pm 1.69 \mathrm{ng} / \mathrm{ml} ; \mathrm{P}=0.070$; Fig. 2).

In MLRs neamine does not affect cell proliferation. In MLRs, neamine did not significantly alter cell proliferation. The proliferation index was $3.54 \pm 0.43$ in untreated MLRs and $3.40 \pm 0.51$ in neamine-treated MLRs ( $\mathrm{P}=0.097$; Fig. 3).

Neamine increases protein levels of Bax and activated caspase-3 in MLR-derived CD4 $4^{+} T$-cells. The expression of $\mathrm{Bcl}-2$, Bax, CC3 and $\beta$-actin in $\mathrm{CD} 4{ }^{+} \mathrm{T}$-cells isolated from the MLRs was assessed by means of western blotting (Fig. 4A) and subsequent quantification of relative protein expression levels (Fig. 4B). In CD4 ${ }^{+}$T-cells isolated from the MLRs, neamine significantly increased the expression of pro-apoptotic Bax by a factor of $2.17 \pm 0.80\left(\mathrm{P}<0.001\right.$ vs. untreated MLR CD4 ${ }^{+}$ T-cells; Fig. 4B). Conversely, neamine had no effect on the expression of anti-apoptotic $\mathrm{Bcl}-2$, altering expression by a factor of $0.96 \pm 0.13$ ( $\mathrm{P}=0.397$ vs. untreated MLR; Fig. 4B). Neamine significantly increased the protein levels of CC3 by a factor of $1.49 \pm 0.13$ relative to untreated MLR CD4 ${ }^{+} \mathrm{T}$-cells $(\mathrm{P}<0.001$; Fig. 4B).

\section{Discussion}

In the present study, the effect of angiogenin on human alloreactive $\mathrm{CD} 4^{+} \mathrm{T}$-cell proliferation and apoptosis was evaluated. 


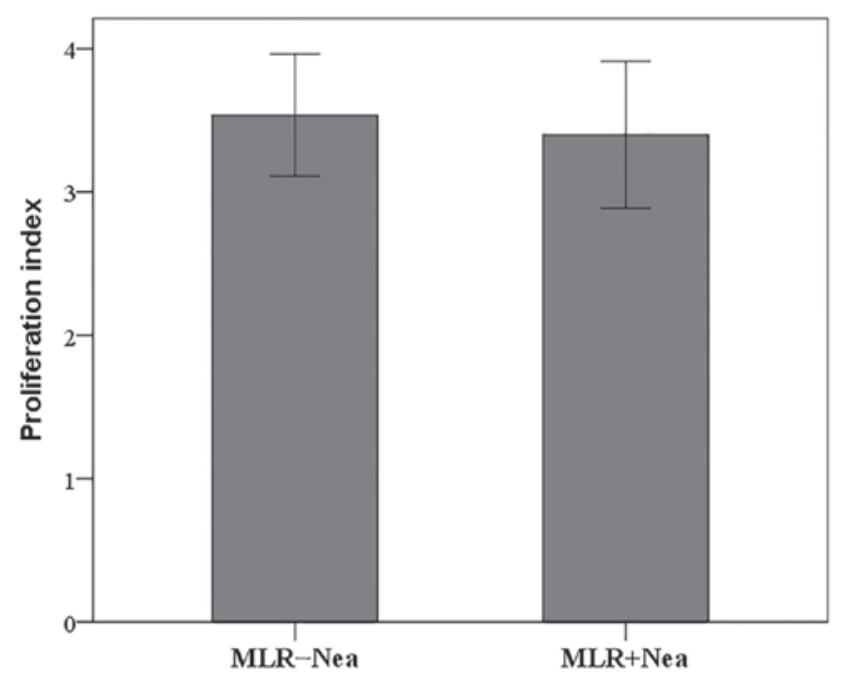

Figure 3. Effect of Nea on cell proliferation in MLRs. Nea did not affect proliferation index in MLRs. Ten different MLRs were performed. Results are presented as the mean \pm standard deviation of triplicate readings. MLR, mixed lymphocyte reaction; Nea, neamine.

For this purpose, the two-way MLR was used as a measure of alloreactivity (15), along with neamine (an angiogenin nuclear translocation inhibitor) treatment (2,16-18). At $100 \mu \mathrm{M}$ neamine did not induce cell necrosis in resting PBMCs or MLRs.

Interestingly, angiogenin production in the supernatants of untreated MLRs was increased by $\sim 1,250 \%$ compared with resting PBMCs controls. This indicates that angiogenin expression increases during the adaptive immune response, which from a teleological point of view makes its potential role in the regulation of the immune response plausible. The presence of neamine did not affect angiogenin production in the MLRs.

Besides the known effect of angiogenin in rRNA transcription, global protein synthesis and cell proliferation $(1,2)$, the angiogenin nuclear translocator inhibitor neamine did not affect cell proliferation in MLRs. Although suppression of cell proliferation by neamine is typical in various cancer cell lines tested $(1,2,16-18)$, this effect is not universal. For instance, neamine inhibits nuclear translocation of angiogenin in both HSC-2 and SAS cells, but proliferation is only suppressed in HSC-2 cells lines (17). This has been attributed to the different expression levels of angiogenin in different cell lines (17). In addition, previous studies have shown that the tiRNAs produced by cytoplasmic angiogenin inhibit global protein synthesis by $20 \%(4,5)$. Such a modest decrease in global protein synthesis may not be adequate to suppress cell proliferation in neamine-treated MLRs.

The results of the current study indicate that angiogenin protects MLR-derived CD4 ${ }^{+} \mathrm{T}$-cells from apoptosis, since neamine, its nuclear translocation inhibitor, increased the protein levels of $\mathrm{CC} 3$, the terminal caspase that all apoptotic pathways converge with (20). Preferential translation of IRES-containing mRNAs is unlikely to be the reason, since Bcl-2 expression remained unaffected by neamine treatment of the MLR-derived CD4 ${ }^{+}$T-cells. Bcl-2 is an anti-apoptotic factor for which translation is mediated via IRES during cell stress (21). Interestingly,
A
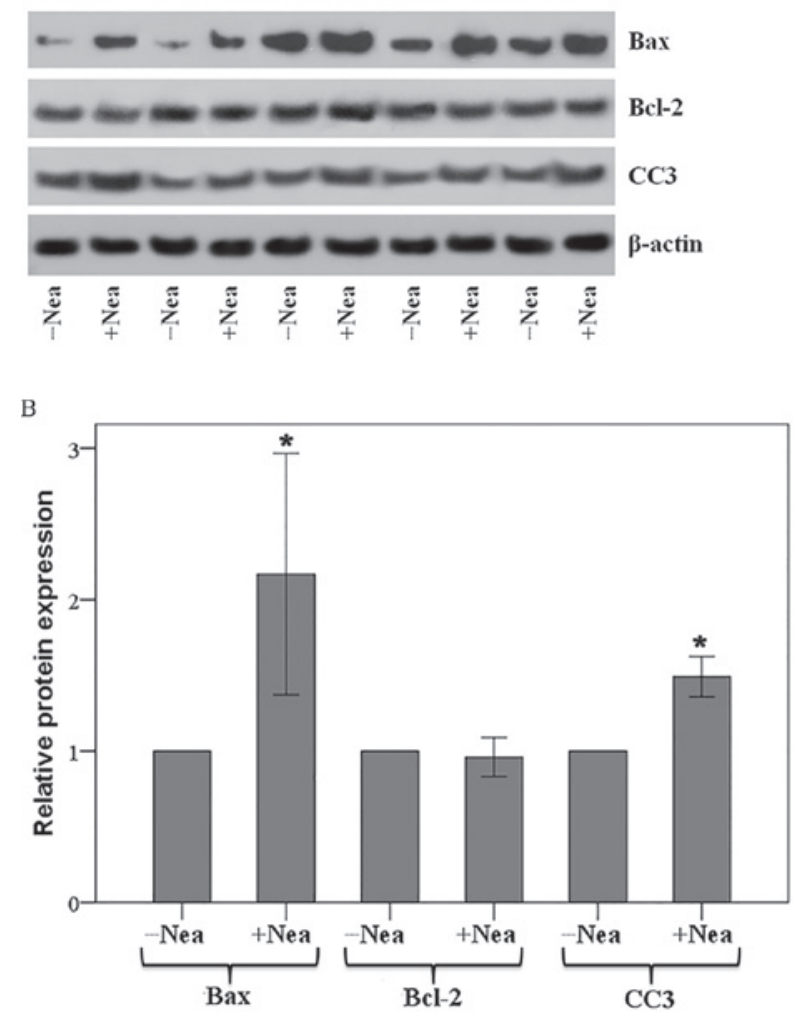

Figure 4. The effect of Nea on the expression of Bax and Bcl-2, and protein levels of CC3 in MLR-derived CD4 ${ }^{+}$T-cells. (A) Western blot of expression levels in MLR-derived CD4 ${ }^{+}$T-cells. (B) Quantification of relative protein expression from western blots in MLR-derived CD4 ${ }^{+} \mathrm{T}$-cells. Expression of pro-apoptotic Bax and protein levels of activated CC3 were significantly increased in $\mathrm{CD}^{+} \mathrm{T}$-cells isolated from MLRs treated with Nea, whereas expression of anti-apoptotic Bcl-2 remained unaffected. Ten different MLRs were performed. Results are presented as the mean \pm standard deviation. ${ }^{*} \mathrm{P}<0.001$ vs. untreated MLR. MLR, mixed lymphocyte reaction; Nea, neamine; Bax, Bcl-2-associated X protein; Bcl-2, B-cell lymphoma 2; CC3, cleaved caspase-3.

a previous study identified that angiogenin-induced tiRNAs did not affect Bcl-2 expression in cells under osmotic stress (9). In that study, tiRNAs protected cells from apoptosis by binding to the cytochrome $c$ released from mitochondria, preventing its binding to apoptotic protease activating factor 1 and apoptosome formation (9), a concept not evaluated in the present study.

In MLR-derived CD4 ${ }^{+}$T-cells, neamine significantly increased the expression of Bax. This suggests increased apoptosis, since the ratio of pro-apoptotic Bax to anti-apoptotic Bcl-2 governs mitochondrial membrane permeability and the release of cytochrome $c$ (20). It is likely that inhibition of angiogenin nuclear translocation by neamine prevents the direct interaction of angiogenin with tumor suppressor p53. This interaction is known to take place in the nucleus and inhibits phosphorylation of p53, increasing p53 binding with mouse double minute 2 homolog and p53 degradation (8). In the absence of nuclear angiogenin, such as when neamine is used, p53 accumulates and increases the expression of its pro-apoptotic transcriptional targets, including Bax, leading to apoptosis (8). A recent study found that in T-cells isolated from MLRs conducted under the same conditions as the present study, p53 and phosphorylated p53 were robustly expressed (22). 
In conclusion, the results of the present study show that angiogenin is upregulated during the alloreactive adaptive immune response. Angiogenin was determined not to effect the T-cell expansion phase, but did inhibit the T-cell contraction phase through reducing $\mathrm{CD} 4^{+} \mathrm{T}$-cell apoptosis.

\section{References}

1. Tsuji T, Sun Y, Kishimoto K, Olson KA, Liu S, Hirukawa S and $\mathrm{Hu}$ GF: Angiogenin is translocated to the nucleus of HeLa cells and is involved in ribosomal RNA transcription and cell proliferation. Cancer Res 65: 1352-1360, 2005.

2. Hirukawa S, Olson KA, Tsuji T and Hu GF: Neamine inhibits xenografic human tumor growth and angiogenesis in athymic mice. Clin Cancer Res 11: 8745-8752, 2005.

3. Fu H, Feng J, Liu Q, Sun F, Tie Y, Zhu J, Xing R, Sun Z and Zheng X: Stress induces tRNA cleavage by angiogenin in mammalian cells. FEBS Lett 583: 437-442, 2009.

4. Yamasaki S, Ivanov P, Hu GF and Anderson P: Angiogenin cleaves tRNA and promotes stress-induced translational repression. J Cell Biol 185: 35-42, 2009.

5. Emara MM, Ivanov P, Hickman T, Dawra N, Tisdale S, Kedersha N, Hu GF and Anderson P: Angiogenin-induced tRNA-derived stress-induced RNAs promote stress-induced stress granule assembly. J Biol Chem 285: 10959-10968, 2010

6. Li S and Hu GF: Emerging role of angiogenin in stress response and cell survival under adverse conditions. J Cell Physiol 227: 2822-2826, 2012.

7. Saikia $M$ and Hatzoglou M: The Many Virtues of tRNA-derived Stress-induced RNAs (tiRNAs): Discovering novel mechanisms of stress response and effect on human health. J Biol Chem 290: 29761-29768, 2015.

8. Sadagopan S, Veettil MV, Chakraborty S, Sharma-Walia N, Paudel N, Bottero V and Chandran B: Angiogenin functionally interacts with p53 and regulates p53-mediated apoptosis and cell survival. Oncogene 31: 4835-4847, 2012

9. Saikia M, Jobava R, Parisien M, Putnam A, Krokowski D, Gao XH, Guan BJ, Yuan Y, Jankowsky E, Feng Z, et al: Angiogenin-cleaved tRNA halves interact with cytochrome $c$, protecting cells from apoptosis during osmotic stress. Mol Cell Biol 34: 2450-2463, 2014.

10. Rybak SM, Fett JW, Yao QZ and Vallee BL: Angiogenin mRNA in human tumor and normal cells. Biochem Biophys Res Commun 146: 1240-1248, 1987.
11. Olson KA, Verselis SJ and Fett JW: Angiogenin is regulated in vivo as an acute phase protein. Biochem Biophys Res Commun 242: 480-483, 1998

12. Eleftheriadis T, Antoniadi G, Liakopoulos V, Pissas G, Stefanidis I and Galaktidou G: Plasma angiogenin and vascular endothelial growth factor a among hemodialysis patients. Iran J Kidney Dis 6: 209-215, 2012.

13. Lioté F, Champy R, Moenner M, Boval-Boizard B and Badet J: Elevated angiogenin levels in synovial fluid from patients with inflammatory arthritis and secretion of angiogenin by cultured synovial fibroblasts. Clin Exp Immunol 132: 163-168, 2003.

14. Brenner D, Krammer PH and Arnold R: Concepts of activated T cell death. Crit Rev Oncol Hematol 66: 52-64, 2008.

15. Sato T, Deiwick A, Raddatz G, Koyama K and Schlitt HJ: Interactions of allogeneic human mononuclear cells in the two-way mixed leucocyte culture (MLC): Influence of cell numbers, subpopulations and cyclosporin. Clin Exp Immunol 115: 301-308, 1999.

16. Ibaragi S, Yoshioka N, Li S, Hu MG, Hirukawa S, Sadow PM and Hu GF: Neamine inhibits prostate cancer growth by suppressing angiogenin-mediated rRNA transcription. Clin Cancer Res 15: 1981-1988, 2009.

17. Kishimoto K, Yoshida S, Ibaragi S, Yoshioka N, Hu GF and Sasaki A: Neamine inhibits oral cancer progression by suppressing angiogenin-mediated angiogenesis and cancer cell proliferation. Anticancer Res 34: 2113-2121, 2014.

18. Liu YP, Hu GF and Wu YX: Neamine is preferential as an anti-prostate cancer reagent by inhibiting cell proliferation and angiogenesis, with lower toxicity than cis-platinum. Oncol Lett 10: 137-142, 2015.

19. Wu D, Yu W, Kishikawa H, Folkerth RD, Iafrate AJ, Shen Y, Xin W, Sims K and Hu GF: Angiogenin loss-of-function mutations in amyotrophic lateral sclerosis. Ann Neurol 62: 609-617, 2007.

20. Fadeel B and Orrenius S: Apoptosis: A basic biological phenomenon with wide-ranging implications in human disease. J Intern Med 258: 479-517, 2005.

21. Sherrill KW, Byrd MP, Van Eden ME and Lloyd RE: BCL-2 translation is mediated via internal ribosome entry during cell stress. J Biol Chem 279: 29066-29074, 2004.

22. Eleftheriadis T, Pissas G, Antoniadi G, Spanoulis A, Liakopoulos V and Stefanidis I: Indoleamine 2,3-dioxygenase increases p53 levels in alloreactive human T cells, and both indoleamine 2,3-dioxygenase and p53 suppress glucose uptake, glycolysis and proliferation. Int Immunol 26: 673-684, 2014. 